\title{
Working with practitioners: how scientists can develop effective relationships that produce an enduring legacy
}

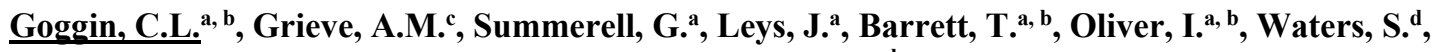 \\ Littleboy, M. ${ }^{\text {, Auld, T. }}{ }^{\text {a }}$ Drielsma, M. ${ }^{\text {a, b }}$ and Jenkins, B. ${ }^{a}$ \\ ${ }^{a}$ New South Wales Office of Environment and Heritage, Australia \\ Email: louise.goggin@environment.nsw.gov.au \\ ${ }^{b}$ School of Rural and Environmental Science, University of New England, Armidale, Australia \\ ${ }^{c}$ Scientific and Environmental Services, Australia \\ ${ }^{d}$ Twyfords Consulting
}

\begin{abstract}
Protecting the environment requires scientists to work with a range of people including land managers, planners, policy makers and regulators. The literature abounds with the challenges of the different 'cultures' working together effectively so scientific results are translated into policy and practice. To address these challenges, we wanted to document factors that produce successful partnerships between scientists and practitioners from different cultures, and determine which factors were the most important in producing an enduring legacy.
\end{abstract}

We asked 12 environmental scientists from the New South Wales Office of Environment and Heritage (OEH) to nominate practitioners with whom they had developed a successful partnership that they believed delivered results for the environment. The scientists nominated land managers, park rangers, scientists, and planners from natural resource management agencies. The projects they were involved with ranged from: protection of threatened species and biodiversity; eradication of feral pests; environmental monitoring; land use planning and management; and planning for climate change.

The scientists each interviewed one of the 12 nominated practitioners (but not the person they nominated). At least one observer was present in all interviews. We used a structured set of questions and asked the practitioner to describe the work and its legacy. We also asked about the processes and systems which fostered or hindered the use of scientific input or expertise in the work; and how the approach and personal attributes of the scientist influenced the outcome. We recorded all interviews which were transcribed then analysed to identify common themes. We found the themes separated into those about the process of working together (nine common themes) and those related to the personal attributes of the scientist (nine common themes).

The common factors mentioned by practitioners about the process of working together were: a collaborative approach; a practical solution; rigorous process that could withstand scrutiny; regular communication; a team with diverse and complementary skills and experience; a shared purpose or vision; being involved from inception; debating how to solve the problem; and an innovative, new or bold approach.

Practitioners mentioned nine common personal attributes of the scientist: a committed, dedicated and passionate person; a leader or champion; a rigorous expert; well connected to universities or other organisations; a clear and effective communicator; who understood the practitioner's aims, needs and constraints; who was accessible and flexible; could function as a knowledge broker and translate complex and technical information into simple terms; and was pleasant, personable and "easy to get along with."

These factors created effective working relationships between scientists and practitioners, and helped bridge the gap between 'cultures.' Our preliminary results, if confirmed by future research, have implications for scientists as well as the organisations that employ them. We suggest ways to foster the common factors mentioned by practitioners so scientists can create more effective working relationships with them. Successful partnerships benefit the scientist and practitioner, as well as the environment.

Keywords: Scientist, collaboration, practitioner, transdisciplinary 
Goggin et al., Working with practitioners: how scientists can develop effective relationships that produce an enduring legacy

\section{INTRODUCTION}

Scientists need to work with people from different 'cultures' including land managers, policy makers and regulators to ensure scientific evidence is translated into policy and practice. However, the literature abounds with the challenges of different 'cultures' working together effectively (e.g. Briggs 2006, Thompson 2009). Indeed, Cullen (1990) suggested there was a 'turbulent boundary' between scientists, policy makers and managers caused by a lack of understanding of the different cultures. The turbulent boundary inhibits information and knowledge being shared between cultures, and affects the translation of scientific results into policy and practice (Hardaker et al. 2009, Ryder et al. 2010, Fazey et al. 2012).

Scientists from the New South Wales (NSW) Office of Environment and Heritage (OEH) are employed to provide evidence and advice to support government policy and management decisions which include protecting the NSW environment. We wanted to improve the likelihood of scientific results from OEH being used in policy and practice by documenting factors that produce successful partnerships between scientists and those from different backgrounds and perspectives. We also wanted to determine which factors were most important in allowing the partnership to produce an enduring legacy for both the environment and those involved.

\section{METHODS}

We asked 12 environmental scientists from the NSW Office of Environment and Heritage (OEH) to nominate a practitioner with whom they believed they had undertaken a successful project that left an enduring legacy. They nominated six practitioners from external organisations: a planner from ACT Government; one scientist from CSIRO and one from the NSW Government; and bureaucrats from natural resource management agencies from the Australian $(n=1)$, Victorian $(n=1)$ and NSW $(n=1)$ governments. They also nominated six OEH staff: one laboratory manager from Science Division, two community engagement staff from Regional Operations Group, and one ranger and two park managers from National Parks and Wildlife Service (NPWS). The projects they were involved with included: protection of biodiversity and threatened species; eradication of feral pests; environmental monitoring; land use planning and management; and planning for climate change.

Each scientist interviewed one nominated practitioner (but not the person they nominated). Most interviews $(\mathrm{n}=9)$ were done by phone, with three done in person. One observer was present in all interviews, with two observers present at nine of the interviews. All interviews took place between August and October 2014, and lasted between 38 and 61 minutes. We used a structured set of 10 questions. In each interview, we asked the practitioner to describe the work and its legacy. We also asked about the processes and systems which fostered or hindered the use of scientific input or expertise in the work; and how the approach or personal attributes of the scientist influenced the outcome.

Interviews were recorded then transcribed by a commercial company with transcripts given to the interviewer and both observers. The transcripts were analysed without the use of computer software to identify common themes (Spradley 1979) and compile a list of the outcomes mentioned by practitioners. The observers and 10 scientists met at a workshop to discuss common themes and their implications for working with practitioners, and gave feedback on being involved in designing the study and undertaking an interview.

\section{RESULTS}

The most common factors mentioned by practitioners in the interviews were categorised into those related to the 'process' of working together, and those related to the personal attributes of the scientist.

The nine most common process factors mentioned by practitioners (in descending order) were:

- Collaborative work/ process (mentioned in 10 interviews, 36 times)

- Practical solution/ application of science: realistic and achievable/ useful (mentioned in 9 interviews, 16 times)

- Rigour/ stands up to scrutiny/ quality assurance (mentioned in 8 interviews, 20 times)

- Regular communication between team (mentioned in 8 interviews, 13 times)

- Diverse/ complementary team/ skills/ experience (mentioned in 8 interviews, 12 times)

- Shared purpose/ vision/goal (mentioned in 6 interviews, 10 times)

- Involve from inception (mentioned in 5 interviews, 9 times)

- Debate how to solve problem/ co-design solution (mentioned in 6 interviews, 8 times)

- Innovative/ new/ bold approach (mentioned in 5 interviews, 7 times).

The nine most common personal attributes mentioned by practitioners (in descending order) were: 
Goggin et al., Working with practitioners: how scientists can develop effective relationships that produce an enduring legacy

- Committed/ dedicated/strong drive/ very keen/ hard worker/ passionate/ enthusiastic (mentioned in 9 interviews, 15 times)

- Leader/ initiator/ champion (mentioned in 8 interviews, 15 times)

- Well connected (to universities for students and expert advice, OEH, Local Land Services, practitioners) (mentioned in 7 interviews, 8 times)

- Expert/ rigorous/ respected scientist/ technical skills/ publishes in scientific literature (mentioned in 7 interviews, 17 times)

- Understands practitioner aims/ needs/ constraints (mentioned in 6 interviews, 7 times)

- Clear effective communicator/ability to talk to public/good public speaker (mentioned in 6 interviews, 6 times)

- Accessible/ flexible/ open/ willing to come to us (mentioned in 5 interviews, 10 times)

- Knowledge broker/ communicates across disciplines/ translates technical information so it's easy to understand/synthesises and communicate work (mentioned in 5 interviews, 10 times)

- Easy to get along with/ pleasant engaging/ friendly/ personable (mentioned in 5 interviews, 8 times).

\section{COMMON PROCESS FACTORS MENTIONED BY PRACTITIONERS}

A collaborative approach was the factor mentioned most frequently by practitioners. The approach was based on mutual respect for each other's knowledge and skills. Practitioners said they "bounced ideas off each other", "feed off each other all the time", "worked with X back and forth about how we might go about that" and "use[d] X as a sounding board." The collaborative approach benefits both practitioners and scientists. For example, "it's been an iterative process of us developing more of an understanding of the science and them developing more of an understanding of the environment that we're operating in."

Practitioners wanted practical solutions that were realistic, achievable and useful. One practitioner said, "his approach is about practical application of science, and achieving outcomes in the field that are realistic and achievable and actually contribute to what we're trying to do there."

Practitioners valued the scientific rigour or quality assurance the scientist brought to the process. For example, "we will have a really solid body of information on which to base decisions." Another practitioner said, "What I need to be able to do is give them the work, let them pick it apart, let them give it to consultants and it stands up. So I need a product that actually represents what's happening on the ground, can withstand scrutiny."

Regular communication between the team was mentioned frequently. This included emails, phone calls or meetings. As one practitioner said, "we would just call each other for anything, anything that really needed other people's involvement or when a decision had been made...X and I pretty much just kept calling each other and there's about 40,000 emails as well."

Practitioners said having a team with complementary and diverse skills created successful working relationships. For example, "it's very complementary.... we're all comfortable with what our individual responsibilities are, and how we interact." Another said, "we're all working together, doing slightly different bits of the jigsaw so that there is actually a picture there which most of us actually understand. We're working on different bits of it and we do our best to fit them together." Another said, "it was just good team work.... he dealt with the science and I dealt with the people." While another said, "we work really well together because he knows what's going on at the state level and I guess I know what's going on at the national level, and we work together to make the most of any opportunities that might be available."

Practitioners also mentioned the importance of a shared purpose, vision or goal to foster effective working relationships. For example, "I've got to say it's pretty well a shared vision, I don't think there's been any conflict about our objectives and priorities." Another said, "X and I had a similar vision to where it could go and he might have known the technical side of it and I might have known how it could work on the ground.

Being involved from inception of a project was important to practitioners. For example, "having X and his team work with us from the beginning to make sure .... everybody's needs were being met, has been terrific."

Practitioners valued being able to debate how to solve the problem and co-design the solution with scientists. For example, "partly because of .... my involvement right from the outset, I was able to work with them in helping to define the problem as well as defining the solution and the specifications that they would work to, to help resolve the problem so we ended up with a much better outcome."

Practitioners also valued an innovative, new or bold approach to their problem. For example, "X was pretty instrumental in pushing along quite a difficult process as it turned out to be, it was quite a big learning process for everyone involved because it hadn't really been done before." 
Goggin et al., Working with practitioners: how scientists can develop effective relationships that produce an enduring legacy

Other factors mentioned by more than one practitioner about the process (in descending order) were: continuity of committed people involved; trust; shared perspective or world view and/or similar styles; working together to define the problem (co-defining the problem or challenge to be addressed); a bespoke solution; that reflected reality or validated their understanding; clear structured approach; flexible or adaptive system.

A collaborative and respectful relationship fosters trust between the practitioner and scientist, which needs time to develop. For example, "what works has come about as a result of the length of the association and number of collaborative activities that I've been involved in with that group."

Factors about the process which practitioners said hindered the outcomes of the work (in descending order) included: budget constraints (including travel and publishing costs); short timeframes to deliver results; IT systems (which block some collaborators and do not allow transfer of large data); geography (including remote locations for research, or geographically dispersed teams); and different operating timeframes (of practitioners and scientists).

\section{COMMON PERSONAL ATTRIBUTES OF THE SCIENTIST MENTIONED BY PRACTITIONERS}

A frequently mentioned personal attribute of the scientist was their commitment, dedication and drive. Practitioners said a scientist who was passionate and enthusiastic ensured the work was done and produced an enduring legacy. For example, "he was excited about the project .... always chomping at the bit to get out there and do stuff, so it was like nothing was too much [trouble]."

Practitioners valued a scientist who initiated, led and championed the work. One practitioner said, "if we'd been asked to do it without assistance from X, it would have been a priority for us but it just would have fallen through the cracks." Another said, "I just don't think it would've happened if X wasn't there."

Scientists with strong professional networks who were "well-connected" were valued by practitioners because they brought additional assets to the work. For example, "his connections to universities and bringing in $\mathrm{PhD}$ students and other researcher that can contribute toward the process and provide expert advice. So I think that's been an important aspect of his involvement." Another said, "I rely on X to deal with [the contracts, systems], so that I don't have to ... he knows the systems, he knows the processes, he knows the organisation at that level much better than I do...That is of enormous value to me."

It is no surprise that practitioners valued scientists as experts who were rigorous and respected in their field with strong technical skills, who published in scientific literature.

Scientists also needed to understand practitioner's aims, needs and constraints. For example, "Understanding what I wanted was the key thing, and tweaking the product like he did. He gave similar products to [others] but ours was unique in some ways and that uniqueness reflected my needs." Another said, "he fundamentally gets what he's got to deliver so the science is very well thought out but very practically oriented."

Practitioners said clear and effective communication was a crucial factor in creating a successful partnership. This included the ability to speak to the public about their work. For example, "X is an extremely good educator and very good public speaker too so I used his skills in that to basically buddy up and partner ... all of those junior staff to pull them up to speed."

Practitioners mentioned that scientists who were accessible/ flexible/ open and willing to come to them, helped create an effective working relationship. One practitioner said, "flexibility to be able to service us in a form that suited us that was a strength from our point of view."

Practitioners valued scientists who functioned as 'knowledge brokers' between different disciplines, synthesising and communicating work, and translating technical information so it was easy to understand. For example, "we have three groups of people in totally different disciplines that really had completely different languages and processes in place and there had to be those brokers ... that kind of mediated between those groups..... X was a knowledge broker between [them]." Another practitioner said "he was good at speaking at their level and translating the science jargon into easily understood information," while another said "he is so good at explaining the really technical side of data transfer, delivery, testing sort of stuff."

By speaking plainly in simple terms, scientists improved the knowledge and understanding of the practitioner and their team and left an enduring legacy. For example, one practitioner said, "I learnt the really .......technical side of it, X was able to clue up a lot of people on it." Another said, "he's just really clear, and he's really calm and he knows the right way of explaining things. He's a good teacher."

Practitioners also mentioned the importance of the scientist being "easy to get along with", pleasant, engaging, friendly and personable. For example, "I can't speak highly enough of X and his team. They've done a 
Goggin et al., Working with practitioners: how scientists can develop effective relationships that produce an enduring legacy

wonderful job and they're really easy, pleasant nice people to work with. Nothing is ever a problem, which certainly makes my life very easy."

In addition to the nine most common factors about the personal attributes of the scientist mentioned by practitioners, other factors mentioned by more than one practitioner (in descending order) were: a scientist who was practical; an educator; with a strategic view; a depth of knowledge and experience; who was reliable and delivered on milestones; encouraged innovation; checked understanding and sought feedback; experienced in field work and/or with users; understands systems and processes in the organisation; could seek funds; was responsive; diplomatic; and "unflappable" and calm.

A lack of time was the only attribute of the scientist which was mentioned by more than one practitioner and hindered the outcome.

\section{LEGACY OF WORK}

Our results indicate a more enduring legacy for the practitioner, scientist and the environment is achieved when a collaborative and rigorous approach is used to create a practical solution.

Practitioners said the legacy of work for the environment included: protecting threatened species; restoring and conserving biodiversity; better planning for climate change; better land management; better management of National Parks in NSW including weed eradication and more visitors with different demographics.

Working with scientists also benefited practitioners and their organisations. Benefits included: value for money; improved ability to prioritise investment; rigorous evidence for decision making; and more confidence in information received. Practitioners also reported increased capability and knowledge after working with scientists. For example, "It's not just bringing someone in, giving the results and leaving. It's about building everyone's capacity because that gives a better outcome." Another said, "I think it was a testament to him that I have high competence in using the data and explaining it because that's certainly not a natural skill of mine." Another said, "I was a bit of a sponge throughout the whole project, just soaking up information from him. .... I know so much more about these [areas] now than anyone has ever known about before, which has helped management [of the National Park] greatly." Another said, "it was very much a two way process, I learnt a lot from him."

The scientist also benefited by gaining a practical grounding for their work. As one practitioner said, “...we were able to offer some good practical grounding for the processes they had in place." Scientists also benefited by gaining a better understanding of the practitioner's environment. For example, "from what I can see, the guys in science are used to dealing with long timeframes and they have time to really ruminate over things and consider them and come up with different iterations. Where in my world, I have 24 hour turnaround times. So it's that difference I guess in operating environments, where sometimes I've really had to push the science guys for information and advice so that I can meet deadlines." Another benefit for scientists was the increased likelihood of repeat business. As one practitioner said "if you trust a person, you'll always go back to them." Another said, "I think that's another thing that's happened as a result of the project. My interest in research has increased and I'm willing to try and facilitate as much as possible other research [here] if I can." To understand the full benefits for scientists, we need to interview them about their experience of working with practitioners.

Scientists who interviewed practitioners in this study also reported several benefits. Similar to practitioners, they valued being involved from inception so they could co-design the work. They also said they enjoyed participating in the interviews and learnt how other scientists worked with practitioners and what practitioners most valued in the working relationships. The scientists also appreciated receiving feedback on their own and other projects (and in some cases, were very pleased to learn their work had a long-lasting legacy). The organisation was also able to collect evidence of outcomes.

\section{DISCUSSION}

The working relationship between the practitioner and scientist was the key to achieving outcomes. As one practitioner said "these projects work really, really well if you develop good working relationships. That was the key to why we had such a long association but also achieved some fairly significant outcomes." Gibbons et al. (2008) also suggested that personal relationships are the basis of successful research-policy partnerships.

Practitioners said an effective working relationship with scientists developed through a collaborative approach. Many authors have advocated a collaborative approach to bridge the divide between scientists and managers (Roux et al. 2006); scientists and policy-makers (Briggs, 2006; Van Kammen et al. 2006; Pannell 2008); and science and decision making (Van Winterfeldt, 2013). 
Goggin et al., Working with practitioners: how scientists can develop effective relationships that produce an enduring legacy

A collaborative approach relies on respect and trust. This allows partners in the relationship to listen to what is needed (or can be provided) and truly seek to understand. In our study, this enabled scientists to understand practitioner's aims, needs and constraints; develop shared vision or purpose; and debate how to solve their problem and co-design a solution. Thompson (2009) found practicing trust and listening ('demonstrating presence') was fundamental to build 'collective communication competence' in interdisciplinary teams. The value and benefit of this seemingly simple activity should not be underestimated. Pannell (2008) also suggested that mutual understanding and trust of researchers and policy makers helped them understand each other's perspective and improve the quality of information flowing between them. Cook et al. (2013) noted that to inform management decisions, as well as being salient (relevant and timely) and credible (authoritative, believable, trusted), science must be legitimate (developed by a process that considers the values and perspectives of all relevant actors).

As expected, practitioners value the rigour and quality assurance which scientists bring to their work together. However, more practitioners mentioned the importance of a practical, realistic and achievable approach. This is often where scientists fail; they have been accused of being "impractical and irrelevant" (Gibbons et al. 2008). Our research suggests practical solutions are more likely when scientists and practitioners communicate frequently; are involved from inception; and the work involves a diverse team with complementary skills.

Of most interest but least surprise, is that the scientist's personal attributes affect the use of their expertise and the outcome of the work (Gibbons et al. 2008). Some attributes mentioned are a product of a scientific career. For example, practitioners valued a respected and rigorous scientist who was well-connected and could bring additional resources (such as university students or other experts) to their work. However, they also valued scientists who led the work, were dedicated and hard-working, accessible, flexible, and "easy to get along with." The latter attributes vary between individuals but, if confirmed through additional interviews, should influence organisations when recruiting scientists who are expected to work with practitioners.

Clear and effective communication was crucial in creating effective working relationships between scientists and practitioners. Many authors advocate communication to bridge the divide between different cultures (e.g. Gibbons et al. 2008). Practitioners valued scientists who acted as 'brokers' between different disciplines to improve relationships between teams by increasing understanding, resolving conflict and ultimately improving rigour. Long et al. (2013) also noted "brokerage can mediate and resolve conflict, make advice and knowledge more accessible, and ....produce environments in which collaboration can flourish." Scientists were most effective when they explain complex scientific information in simple terms, using examples and analogies.

Our results indicate a collaborative approach delivers a legacy for the practitioner, the scientist and the environment. Practitioners said that by working with scientists who shared their knowledge openly and respectfully, they increased their capabilities and "learnt a lot." This can also benefit scientists because, according to Eden (2011), the more understanding decision makers gained about research, the better they understood what it could provide, how to communicate their needs and how to make use of results. The outcomes reported by practitioners in our study suggests this is also the case.

\section{CONCLUSION AND RECOMMENDATIONS}

Our preliminary results, if confirmed by future research, have implications for scientists and organisations that employ them.

We found a collaborative approach creates rigorous, practical and useful solutions that benefit practitioners, scientists and the environment. But relationships do not happen overnight: both scientists and practitioners need to invest time so they can understand each other's environments, build trust and collaborate effectively.

We found factors about the 'process' of working together, as well as personal attributes of the scientist affect the relationship. Some 'process' factors can be fostered within organisations by training scientists about bestpractice (i.e. frequent communication, involve practitioners from inception - to define the problem together, debate how to solve it, and co-design a solution). Some personal attributes, such as being an effective communicator and knowledge broker, can also be fostered through training as well as mentoring. The most effective communicators and knowledge brokers also had experience working across cultures, so scientists should be encouraged to work with practitioners from different disciplines to improve their skills.

Some personal attributes mentioned by practitioners in this study will be more difficult to foster. For example, respecting practitioners, and being a leader, as well as flexible, accessible and hard-working, is difficult to teach. However, organisations can reward scientists who exhibit these attributes and recruit scientists who possess them. This would nurture a collaborative culture in the workplace. 
Goggin et al., Working with practitioners: how scientists can develop effective relationships that produce an enduring legacy

Scientists who participated in this study also said they benefited from being involved. We recommend similar interviews at the end of projects so scientists learn from their interactions with practitioners and improve future work. End-of-project interviews could also be used to document outcomes and demonstrate value.

We believe our results apply to many areas of transdisciplinary work where different 'cultures' work together. From these preliminary results, we have drafted a best-practice checklist (which we will present at the conference) to guide work between scientists and practitioners, and other disciplines that work together. In future, we will undertake more interviews to confirm our preliminary results and finalise the checklist. Ultimately, our study will help foster a collaborative and rigorous approach between different cultures that creates practical solutions and benefits practitioners and scientists, and leaves a legacy.

\section{ACKNOWLEDGEMENTS}

We thank practitioners who shared their experience of working with OEH scientists, and scientists who interviewed them. We also thank two anonymous reviewers for their constructive comments.

\section{REFERENCES}

Briggs, S. (2006). Integrating policy and science in natural resources: why so difficult? Ecological Management \& Restoration, 7, 37-39.

Cullen, P. (1990). The turbulent boundary between water scientists and water managers, Freshwater Biology, 24, 201-209.

Fazey, J., Evely, A.C., Reed, M.S., Stringer, L.C., Kruijsen, J., White, P.C.L., Newsham, A., Jin, L., Cortazzi, M., Phillipson, J., Blackstock, K., Entwistle, N., Sheate, W., Armstrong, F., Blackmore, C., Fazey, J., Ingram, J., Gregson, J., Lowe, P., Morton, S., and C. Trevitt (2012). Knowledge exchange: a review and research agenda for environmental management, Environmental Conservation, 40, 19-36.

Gibbons, P., Zammit, C., Youngentob, K., Possingham, H., Lindenmayer, D., Bekessy, S., Burgman, M., Colyvan, M., Considine, M., Felton, A., Hobbs, R., Hurley, K., McAlpine, C., McCarthy, M., Moore, J., Robinson, D., Salt, D., and B. Wintle (2008). Some practical suggestions for improving engagement between researchers and policy-makers in natural resource management. Ecological Management \& Restoration, 9, 182-186.

Hardaker, J.B., Fleming, E., and G. Lien (2009). How should governments make risky policy decisions? Australian Journal of Public Administration, 68, 256-271.

Long, J., Cunningham, F.C., and J. Braithwaite (2013). Bridges, brokers and boundary spanners in collaborative networks: a systematic review, BMC Health Services Research, 13, 158.

Pannell, D. (2008). Engaging with policy: tips for researchers, Pannell discussions, no. 136, http://dpannell.fnas.uwa.edu.au/pd/pd0136.htm

Roux, D.J., Rogers, K.H., Biggs, H.C., Ashton, P.J., and A. Sergeant (2006). Bridging the science-management divide: moving from unidirectional knowledge transfer to knowledge interfacing and sharing, Ecology \& Society, 11, 4 .

Ryder, D.S., Tomlinson, M., Gawne, B., and G. Likens (2010). Defining and using 'best available science': a policy conundrum for the management of aquatic ecosystems, Marine and Freshwater Research, 61, 821-828.

Spradley, J. (1979). The ethnographic interview. Rinehart and Winston, New York.

Thompson, J. (2009). Building collective communication competence in interdisciplinary research teams, Journal of Applied Communication Research, 37, 278-297.

Van Kammen, J., de Savigny, D., and N. Sewankambo (2006). Using knowledge brokering to promote evidence-based policy-making: the need for support structures, Bulletin of the World Health Organization, 84, 608-612.

Von Winterfeldt, D. (2013). Bridging the gap between science and decision making. Proceedings of the National Academy of Science, 110, 14055-14061. 